\title{
Agent-Based System Architecture for Intelligent Lighting Control Based on Resident's Behavior
}

\author{
Agne Paulauskaite-Taraseviciene, Nerijus Morkevicius, Vaidas Jukavicius, Laura Kizauskiene, and \\ Egidijus Kazanavicius
}

\begin{abstract}
-learning from the behavior of the resident is essential in order to adapt the system to provide intelligent lighting control. Technologies to assure two aspects, coordinated system control and intelligent decision making, are required for such system implementation. In this paper an agent-based system architecture that aims to provide intelligent lighting control according to resident's habits is proposed. The multi-agent technology has been integrated with intelligent decision making algorithms seeking to provide fast, adaptive and accurate lighting control. To explore capabilities of the proposed lighting control system, the experiments including resident's changing habits and unpredictable behavior have been performed, evaluating accuracy and speed of control.
\end{abstract}

Index Terms-Agent-based system, intelligent lighting control, behavior patterns, resident habits.

\section{INTRODUCTION}

The understanding of intelligent control is outgrowing the traditional concept for home automation systems, typically acting according to predefined control strategies. Intelligent systems should combine comfort, appliance scheduling and safety constraints with dynamically changeable users' preferences. This means that intelligent control systems are supposed to take into consideration another important issue -the behavior of its users. The growing importance of human-orientation is witnessed by the number of research activities in this field. Activity recognition and analysis frameworks [1], [2], human behavior modelling and pattern extraction models [3], habit profiles [4], [5] are only a few instances of the proposed approaches, leading to contemporary concepts of personalized services', which in turn become key enablers for implementing an important objective of intelligent home systems - maximization of resident's comfort.

Systems, that take into account human behavior aspects, can enhance energy efficiency, saving up to $40 \%$ of home energy resources [6]. In this field the research on intelligent lighting control has become very important. Increasing user's comfort and reducing energy costs have always been two primary objectives of intelligent lighting control systems. It is quite difficult to provide control satisfying level of individual comfort and the energy reduction goals simultaneously. However, for residential homes the individual comfort plays more important role than energy costs. Comfort level of lighting can be indicated by following resident's behavior that would give a double benefit: lighting control based on

Manuscript received July 24, 2014; revised September 29, 2014.

The authors are with the Kaunas University of Technology, Lithuania (e-mail: agne.paulauskaite-taraseviciene@ktu.lt). resident's habits and possibility to avoid unnecessary energy consumption if luminaires are likely to be left on after use or accidentally switched. The main goal of such systems is to anticipate the resident' periodic actions and to help them in daily routines. Another important objective that must be taken into account refers to changing individual habits over the time. Therefore, intelligent system has to be flexible and dynamic and promptly respond to changing and diverse environment. It's always possible to ask the resident for confirmation of its changes wishes, but to make decision after the resident being questioning it's not the best solution for two reasons. First of all, not always it's possible to identify behavior habits for the resident itself, because sometimes habits are changing slowly, inconsistently or for the specific situations. Secondly, intelligent and unobtrusive control system should be able to adapt automatically and make right decisions in changing environment, not asking the questions with requests to approve resident's behavior. Creation of such system according to all stated objectives consequently leads to the implementation of approaches able to follow residents' habits, adapt to changing environment and to promptly provide the control of lighting sources.

\section{InTELLIGENT Lighting CONTROL SYSTEMS}

Most of academic or commercial systems aim to modernize control of lighting in terms of the minimization of energy consumption [7], [8]. Therefore, the major attention is paid to the energy-efficient lighting systems that are able to estimate a sufficient illumination level and control lighting sources based on various sensors [1], or predefined rules, i.e., time and week day conditions [10]. A strong motivation for such systems emerged from business area (typically, in large office buildings) where the illumination constitutes a large proportion of the main energy consumption. But intelligent lighting control system for residential homes should maintain the balance between energy saving and residents' visual or psychological comfort provision assigning a priority to individual habits.

There are plenty of methods helping to deal with adaptive control issues in smart home domain. The usage of such methods as Artificial Neural Networks (ANNs), Fuzzy Logic (FL) or Bayesian classifiers provide expected functionality for intelligent systems through the learning, adaptation or prediction capabilities [11]-[13]. Denoted approaches can suggest the final decision then all required information is reported. The complexity of the problem determines the content of necessary information. Resident's lighting habits may be detected from resident ${ }^{\star} s$ coordinates, pose, local time, ambient light and so on. In the Smart Homes, all required 
information usually is captured by sensors. The system must process a huge amount of information: data acquisition from sensors, status checking of various objects and entities, residents' feedbacks, commands transmission for the actuators and etc. It's obvious that all necessary information must be collected as soon as possible. However, to anticipate the variation of the number of information sources in time increase the system's flexibility. In this context, another artificial intelligence technique, namely multi-agent systems, has gained a wide acceptance and are founded as appropriate or even one of the fundamental technologies for building smart environments.

The distributed nature of intelligent home systems need a total autonomy of its components in order to assure system's robustness, to fulfil time-bounded and space-bounded requirements. Therefore, the integration of agent technology and machine learning methods can give much better synergetic effect, resulting in high degree of autonomy, flexibility, adaptation and personalization of system components that lead to the highest intelligence level of the whole system.

Agent-based researches in smart home environments usually focus on several aspects: the technologies, enabling the automation and optimization of household appliances [14], [15], investigations with respect to inhabitant behaviour [16], researches, covering MAS-based generic architectures and infrastructures, devoted for developing intelligence systems [17]. An agent-based architecture itself provides several of advantages including trainability, efficiency, fault tolerance, extendibility, and scalability. Taking into account these advantages, this paper presents the implementation of intelligent lighting control system using the proposed agent-based architecture highlighting information gathering, processing and communication between agents.

\section{Proposed Multi-Agent System Architecture}

In this research the requirements for multi-agent system be able to control the illumination according to residents' behavior are listed below:

- Intelligent. Learning and re-learning capabilities are required in order to provide the control according residents' habits. System should be able to use important information from environment, including importance evaluation and dependencies determination between data parameters. Besides, it has to be able to adapt according to the changes in the environment or resident's habits by specified adaptation rate.

- Dynamic structure. The number of controllable devices at the residential homes is varying in time, therefore the system should be able automatically to adapt (add/remove required components) and provide a stable performance.

- Autonomic. All agents of controllable devices have to be of the same architecture and to be able to perform as a single unit and as a member of the agents group.

- Available and Reliable. System should be fully distributed in order to avoid a single point of failure.

The basic structure of the proposed multi-agent system architecture for single actuator is presented in Fig. 1. In the architecture three logical layers are specified. The system can fully operate if there is at least one agent in the each of three logical layers. In general, all distributed agents can be hosted in one physical hardware, but such type of integration lacks practical utility. Therefore, all agents are hosted in the different intelligent devices with specific hardware (light sensor, actuator, etc.) which are connected to the LAN. Each intelligent device has the microcontroller running on the independent agent platform (e. g. JADE [18]) that hosts several agents. These agents provide communication and services for the hardware of the intelligent devices.

All devices (facilitating independent agent platforms) run one assistive BDF agent and one or more of common agents providing different services. Some of the common agents are specific for particular physical devices while the others are independent from physical location. For example, Light Sensor Agent can only run on the device which physically has the light sensor. In contrary, the Decision Agent which is responsible for control decisions could be hosted in any physical device. All the intelligent devices communicate between themselves using standard FIPA ACL [19]. This allows avoiding direct communications between physical devices which is expensive and lacks flexibility.

In the proposed architecture four classes of agents are included which are described in details further is this section.

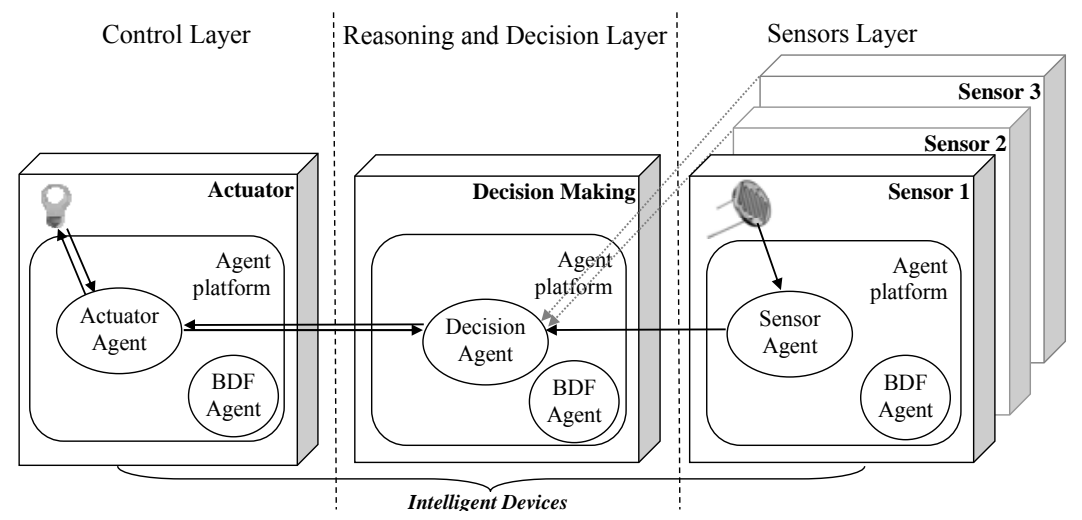

Fig. 1. Distributed agent-based architecture for intelligent control of single actuator.

\section{A. Sensor Agents}

The functions of Sensor Agent (SA) are to monitor readings of the surrounding environment from certain sensors or their groups, to interpret these readings, to evaluate their significance and to inform Decision Agents (DAs) about the changes in the environment if necessary. All SAs have a common internal structure but depending on the sensor type 
they differently process data received from sensors. For lighting control, main SAs are as follows:

Lighting Sensor Agent (LSA). This agent monitors the readings of lighting sensor and decides when to inform DAs about occurred changes in the sensor readings. According to sensor data dynamics, LSA has to distinguish when a significant change of the ambient light has occurred and what had an influence on them. As an example, the readings of lighting sensor can constantly be changing if residents are walking more intensively in a small area and putting a shadow on the sensor. If minimum time limits are set between adjacent changes, LSA ignores these short-term variations in lighting sensor (overshadowing) and does not send any signal for DAs. The prototype of the intelligent light sensor facilitating agent platform with LSA and BDF agents is presented in Fig. 2.

Resident Location Sensor Agent (RLSA). The purpose of this agent is to monitor the location of the resident using hybrid ultrasound and radio frequency (RF) technology. In this case the physical sensor is composed of several beacons and listeners. Each resident carries one personal beacon which emits synchronized (using RF technology) ultrasound signals to the environment. Listeners are located in the specific places of the room and listen to the incoming ultrasound and RF signals. RLSA calculates the exact position of the resident by measuring ultrasound propagation times from the personal beacon to all listeners. If the significant change of resident's coordinates is registered, RLSA sends the corresponding message to Decision Agents.

Two essential parts of the prototype of resident's location sensor are presented in Fig. 2 and Fig. 3.

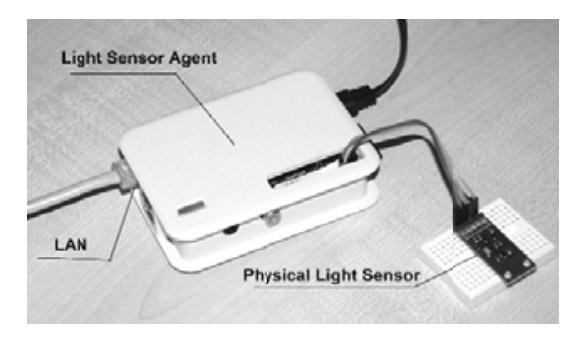

Fig. 2. Prototype of intelligent light sensor.

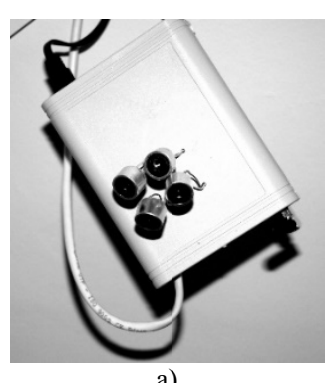

a)

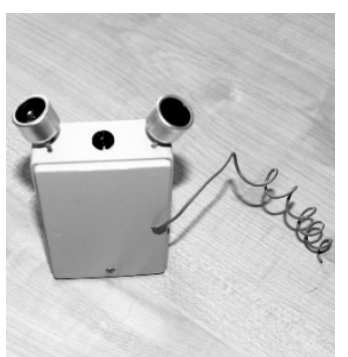

b)
Fig. 3. The main parts of the prototype of resident's location sensor: ultrasound and RF listener a) and personal ultrasound beacon b)

\section{B. Control Agents}

Control Agents (CAs) are responsible for the management of devices situated in the environment by employing actuators. These agents have built-in interface in their structure to control certain actuators. CAs always receive information from their specific DA, which provides a controlling solution. In addition, these agents also monitor the changes in the controlled actuator status. These changes are usually initiated by a resident, who shows that the environment is not comfortable and sets the particular actuator to his desired status. If such event occurs, CA informs DA about new resident's desires and thus causes learning or re-learning procedure. It may be that the resident will be changing the status of actuator too often or unpredictably. Such resident's actions are ignored by CA because the minimum time needed to get the steady status for actuator is set in advance, after which the agent can send a learning signal to Control Agent.

\section{Decision Agents}

The purpose of Decision Agent (DA) is to make decisions under changing environmental parameters. DAs control certain devices located in the environment by using information received from SAs. Each device has one its own DA and CA. The structure of DAs is the same but they can use different algorithms for decision making solutions. DAs operate in two modes:

- Decision making mode is activated when the controlling decision must be provided under certain environmental conditions. The algorithm of decision making for concrete situation is executed based on the information gathered from sensor agents;

- Learning or re-learning mode is activated when already provided solution is adjusted by the resident making settings for the next status of actuator. In this case, the feedback with information for re-learning is sent from Actuator Agent to Decision Agent. However, if the system is in the learning mode it uses all resident's settings needed for the learning.

\section{BDF Agents}

Each intelligent device in the proposed architecture must meet strict requirements of fully distributed architecture. This requirement imposes inability to use standard FIPA Agent Directory Services of the agent platform (e.g. the DF Agent in every JADE platform) in order to find out what other agents are available in the environment. Moreover, some of the devices may constantly appear/disappear (for example, the resident enters and leaves the room). The service agents must have the means to find out what other agents are running at the certain time moment in the whole distributed system of independent agent platforms. To meet these requirements the Biatech Directory Facilitator (BDF) agent was developed.

The main task of the BDF agent is to constantly monitor the changing environment and provide the list of "known" agents to other agents residing on the same platform. For this purpose, the message exchange process between all BDF agents is initiated. All messages sent between agents are the standard FIPA ACL messages.

BDF agent's function from the viewpoint of common agent is very straightforward. For example, if the sensor agent detects a significant change of lighting, it has to send a message to all DAs. To find out the addresses of all DAs running in the whole environment at the current time, the sensor agent sends synchronous message GET_AGENTS with filter to the local BDF agent. BDF agent responds with the list of currently known DA agents (message GET AGENTS RESP). Sequence of message exchange between common agent and local BDF agent is presented in Fig. 4. 
To assure the correct performance of BDF agents, each newly created(born) common agent must register at the local BDF agent, providing the list of services it provides (message AGENT_NEW). Additionally, each common agent sending message AGENT_LIVE must report to BDF agent that it is still running at defined time intervals. BDF agent tracks all the messages from all local agents and removes the agent from the list if it doesn't receive the message during the regular checking with $3 \mathrm{~T}$ time period.

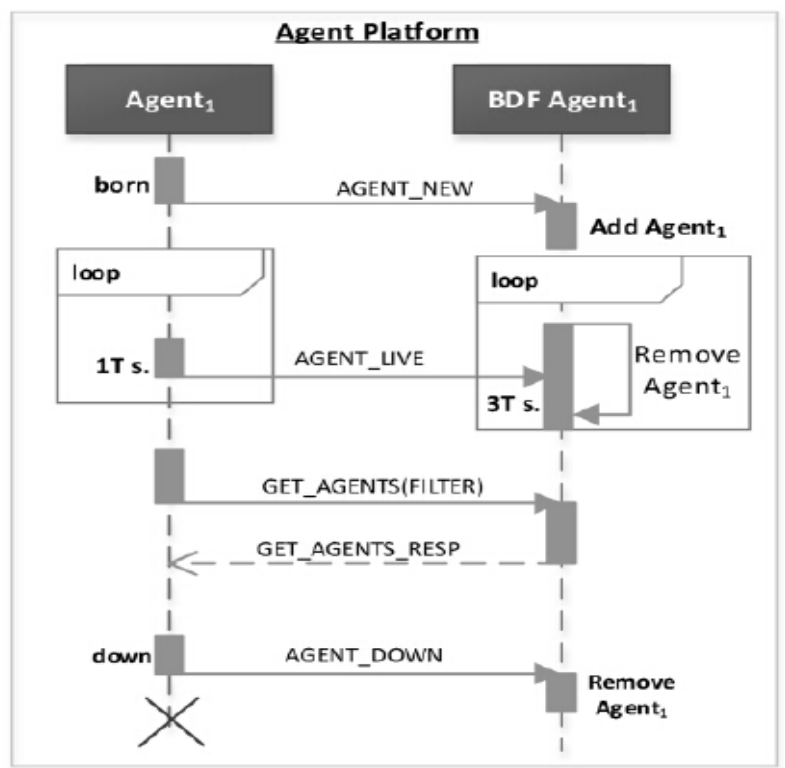

Fig. 4. Sequence of message exchange between common agent and local BDF agent.

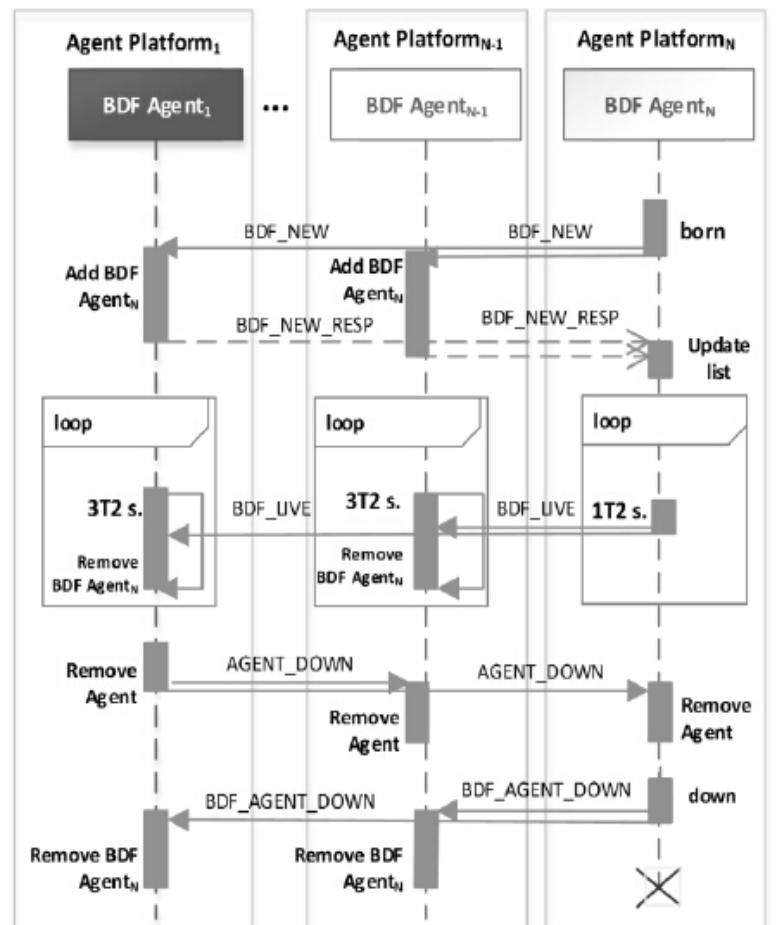

Fig. 5. Sequence of message exchange between BDF agents on different platforms.

The advantage of this architecture is that the common agents communicate only with the local BDF agents; thus reducing time and complexity of communication. Tracking of the current states of all the other agents in the distributed system is achieved by internal BDF agents' communications.
Sequence of message exchange between BDF agents is presented in Fig. 5. BDF agents share information about all critical events (creation and removal of common and BDF agents, etc.) occurring in their local platforms. In such way each BDF agent has information about all the agents in all platforms.

The new created BDF agent finds out the addresses of the other BDF agents using lower level communication protocol. Each BDF agent has its own IP server listening on special multicast address. The new BDF agent uses a multicast protocol and sends a special structure request message to all listening servers. If in the predefined timeout period some responses are received, the BDF agent knows the IP addresses of other BDF agents and can start the standard FIPA ACL message level communication.

The first message is BDF_NEW, which informs all other agents that the new BDF agent is created. In response to this message all remote BDF agents send BDF_NEW_RESP messages containing the list of all known agents. Tracking for removed BDF agents is performed in the similar fashion as tracking for common agents (see Fig. 4).

\section{EXPERIMENTS}

\section{A. Scenario of Lighting Control}

In this research taken example of intelligent environment (enabling to observe residents, collect environmental data and to control lighting) is presented in Fig. 6. The environment consists of intelligent sensors capable to identify residents $\left(I D_{1}\right.$ and $\left.I D_{2}\right)$ and track their position in the room. Lighting conditions near the tables $T_{1}$ and $T_{2}$ are recorded using light sensors $S_{1}$ and $S_{2}$. Dimmable luminaires $L_{1}$ and $L_{2}$ are capable to report their status to the controlling system as well as to adjust their settings in response to the signals received from the controlling system.

The lighting zones, specified in experimental test-bed, define resident's positions and values of particular sensors. Five different zones $L 1, L 2, L 3, L 1-L 2$, and Neutral are excluded. Predefined values of sensors for possible lighting wishes in each zone are provided in Table I.

Lighting A means 220-260 lx illumination by the table. One user (ID1) wants that coming near any table the lamp above him would light up at the required brightness and the sensor located below this lamp should show the desired brightness 220-260 lx.

Lighting B means 300-340 lx illumination by the table. Another user (ID2) wants that coming near any table the lamp above him would light up at the required brightness and the sensor located below this lamp should show the desired brightness 300-340 lx.

Lighting $\mathbf{C}$ is activated if users are receding further from the table and the lamps are switched off, i.e. only the ambient illumination is on.

Lighting D means 160-180 lx illumination between two tables. Lamps must illuminate approximately at the same brightness that both sensors would show 160-180 lx.

The architecture of the actual multi-agent system, which has been used in our experiments, is presented in Fig. 7. LSA is responsible for detection of significant lighting changes. 
Each of the two residents has a personal beacon device. RLSA agent is created each time the resident enters the room. RLSA is responsible for tracking the significant location changes of the resident. These changes are reported to all Decision Agents using FIPA ACL messages.

Two logical agent layers were hosted on the single physical devices for simplification of prototypes. Each dimmable luminaire facilitated two agents. CA is responsible for the adjustment of the intensity of the local luminaire according to the messages received from the corresponding DA. DA employs a particular algorithm to make the control decision of particular luminaire. Three different decision making algorithms based on ANN, Fuzzy Logic and Bayesian approaches are applied in this research for accuracy evaluation.

If the resident isn't satisfied with the current lighting, he manually adjusts the actuator. In such case the local CA sends the message to all DA, telling them to make an adaptation (re-learning) according to newly received information.

All required agents have been implemented using Java and JADE Agent platform. All JADE platforms were running on Raspberry Pi B devices using Linux OS.

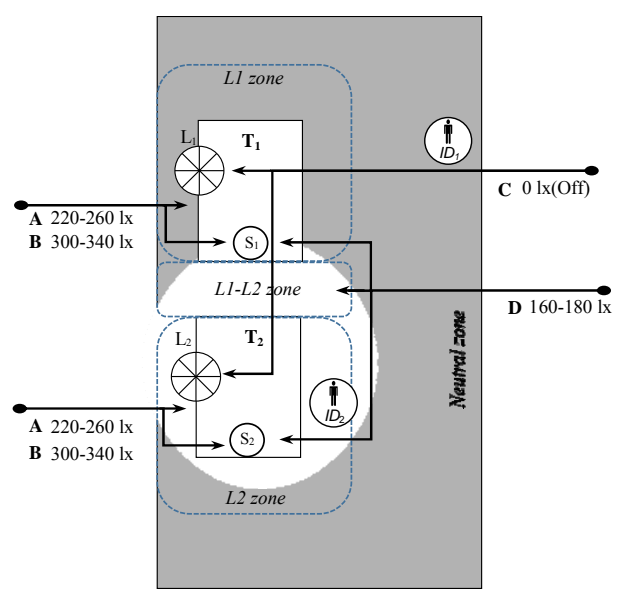

Fig. 6. Test-bed of lighting control.

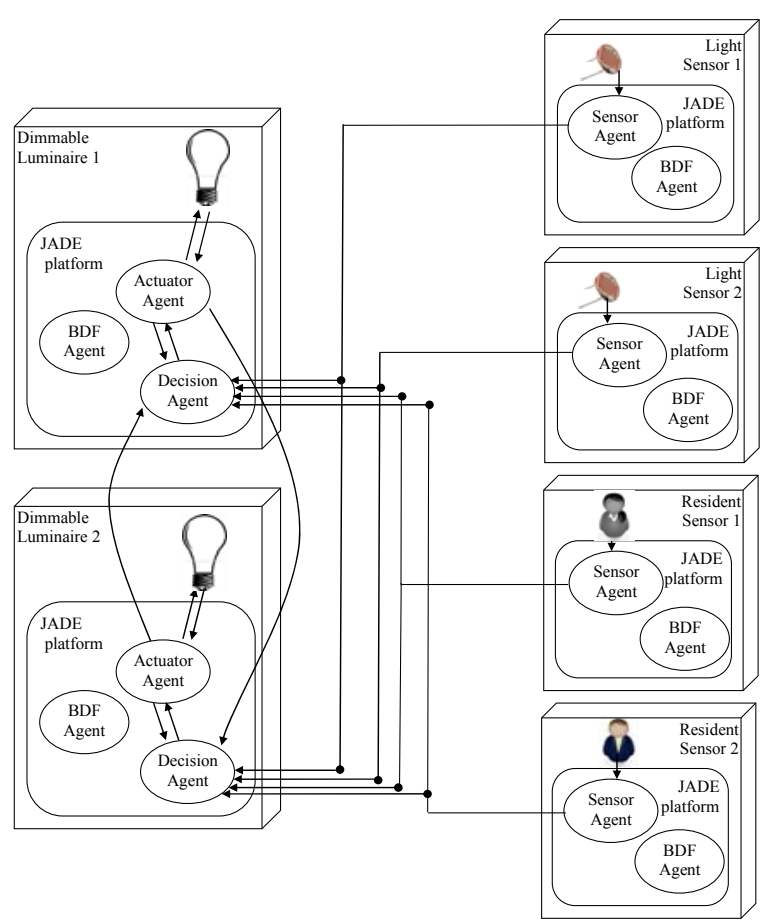

Fig. 7. Agent based architecture for lighting control test bed.
TABLE I: PREDEFINED VALUES OF SENSORS ACCORDING LIGHTING

\begin{tabular}{|c|c|c|c|}
\hline \multicolumn{4}{|c|}{ WISHES } \\
\hline \multirow{4}{*}{$\begin{array}{l}\text { Lighting } \\
\text { A }\end{array}$} & \multirow{4}{*}{$\begin{array}{l}\text { ID zone } \\
\text { L1 } \\
\text { L2 }\end{array}$} & \multirow{3}{*}{$\begin{array}{l}\text { Sensors } \\
\mathrm{S} 1,1 \mathrm{x} \\
220-260\end{array}$} & \multirow{3}{*}{$\mathrm{S} 2,1 \mathrm{x}$} \\
\hline & & & \\
\hline & & & \\
\hline & & & $220-260$ \\
\hline \multirow{2}{*}{ B } & L1 & $300-340$ & \\
\hline & L2 & & $300-340$ \\
\hline $\mathrm{C}$ & neutral & \multicolumn{2}{|c|}{ background lighting } \\
\hline $\mathrm{D}$ & L1-L2 & $160-180$ & $160-180$ \\
\hline
\end{tabular}

\section{B. Experimental Results}

Scenario 1 has been developed in order to determine how accurately the agent system trained in advance according to the wishes of residents is able to provide solutions. The fragment of experimental results under backlight setting 150 lx is given in Table II. Results show that system is able accurately to control the ambient lighting devices if it employs any decision making algorithm.

TABLE II: SCENARIO 1 FRAGMENT OF RESULTS

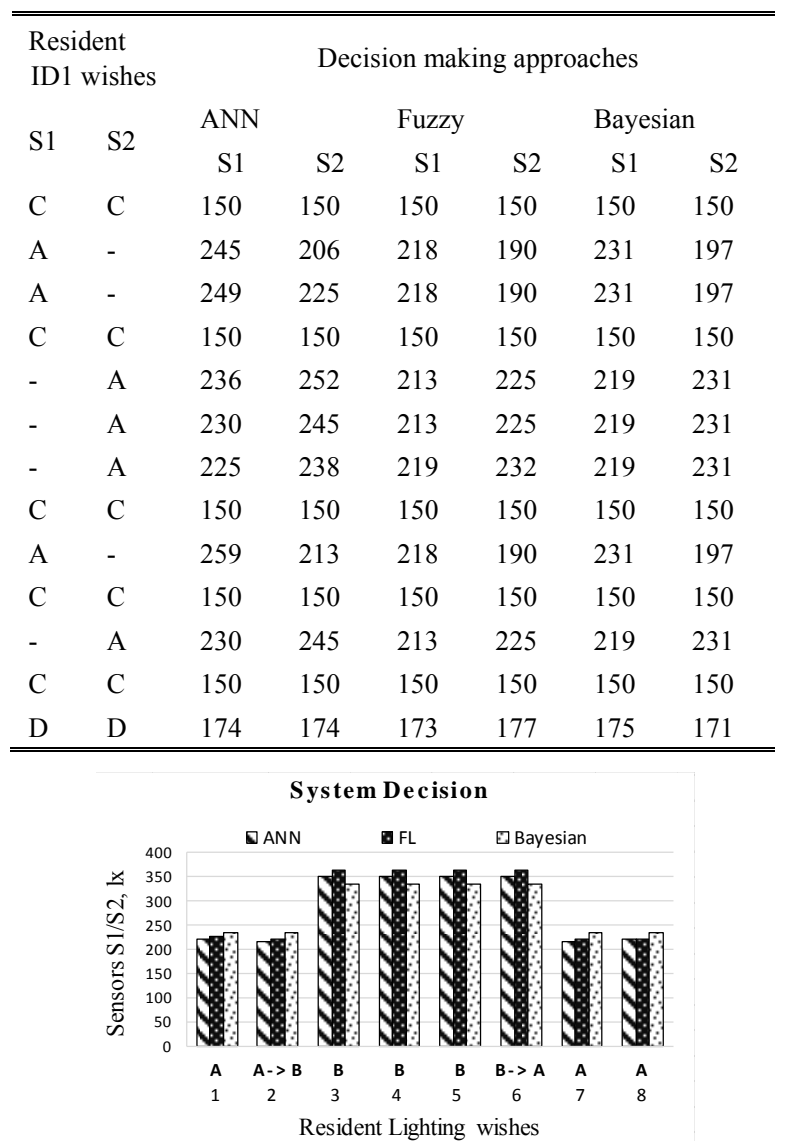

Fig. 8. System lighting decision with 1 step adaptation to new and old resident habits.

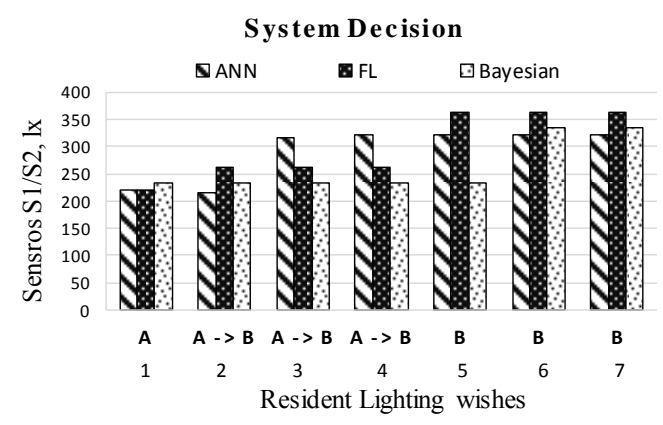

Fig. 9. System lighting decision with 3 steps adaptation to new resident habits. 


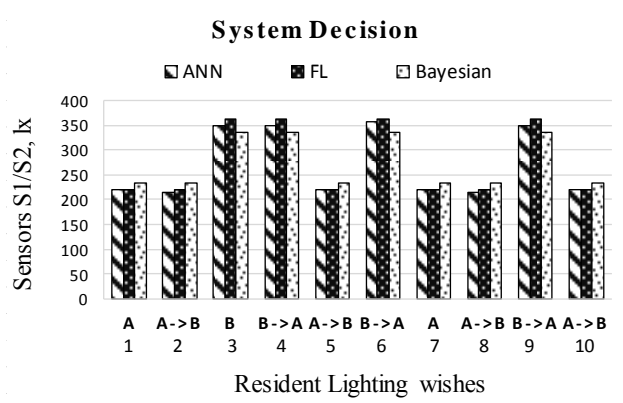

Fig. 10. System lighting decision error with 1 step adaptation to repetitive changes of habits. System Decision

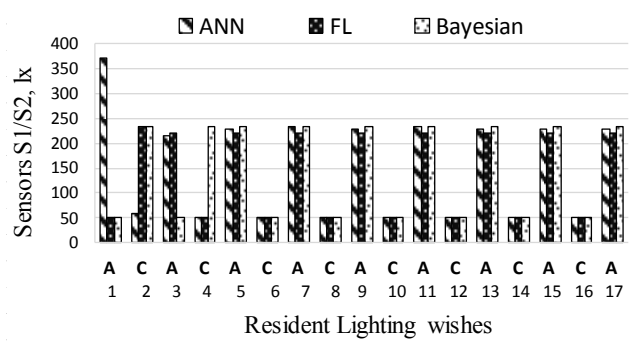

Fig. 11. ANN, FL and Bayesian methods decision error in the initial learning process.

Scenario 2 has been designed to explore how the system is able to respond to a new temporal resident's wish and how quickly adapts if the resident returns to his previous habits. The second step includes resident's wish B, which is temporal; the sixth step shows his choice of previous lighting habits A (Fig. 8). The experimental results infer that system is able to make fast adaptations if resident's habits change in time using any decision making algorithm and to provide solutions according to his last wishes. Fig. 9 presents results showing the obtained solutions with the predefined 3 steps adaptation requirement for the system. The system has to perform slower adaptation and ignore perhaps unintentional or simultaneous actions while be able to react to new changes step by step.

Scenario 3 has been developed to observe how the system is able to respond to those situations in which the resident begins to behave inconsistently and often changes his lighting wishes from A to B and vice versa (Fig. 10).

Scenario 4 has been designed in order to determine how quickly the system is able to learn if there is no information about resident's wishes. In this case, the system is in an initial status and is starting to train. This scenario assumes that resident exactly knows what he wants. The experiment results show that the system can learn and adapt according to the resident's wishes $\mathrm{A}$ and $\mathrm{C}$ after few iterations (see Fig. 11).

TABLE III: LIGHTING CONTROL SPEED

\begin{tabular}{|c|c|c|c|}
\hline \multirow{2}{*}{$\begin{array}{l}\text { Data processing sequence } \\
\text { for lighting control }\end{array}$} & \multicolumn{3}{|c|}{ Mean processing speed, $(\mathrm{ms})$} \\
\hline & Bayesian & ANN & FL \\
\hline 1.Hardware & & 150 & \\
\hline 2.Sensor Agent & & 300 & \\
\hline 3.LAN & & 2.8 & \\
\hline 4.Collecting data & & 150 & \\
\hline 5.Decision making & 9.5 & 6.7 & 60 \\
\hline 6.JADE & & 40 & \\
\hline 7.Actuator Agent & & 3.5 & \\
\hline 8.Dimmable luminaire reaction & & 20 & \\
\hline Total reaction time & 612 & 602 & 662 \\
\hline
\end{tabular}

The results of experimental evaluation of the situation changes' propagation time from intelligent sensor through the distributed control system to the final intelligent actuator are presented in Table 3. The first column in the table shows the whole path of propagation. In the first step the actual physical light sensor detects the changed lighting, then the corresponding LSA evaluates the changes. If the changes are significant, then the corresponding FIPA ACL message is sent to DM agent using LAN. The process follows (see Fig. 7) until the control decision is taken and intensity of the physical dimmable luminaire is adjusted. The second column in the table shows the times taken in each step. The times of decision making step are different depending on used decision making algorithm. Finally the total reaction time is evaluated for each algorithm separately.

\section{CONCLUDING REMARKS}

In this paper agent-based system architecture for intelligent lighting control according to the resident's habits has been proposed implementing two main aspects of behaviour based systems: innovative monitoring of resident's behaviour and intelligent adaptation.

Utilization of agent technology has enabled to make the intelligent monitoring using different devices. The proposed architecture has provided stable, fast and accurate lighting control. In each layer of this architecture more than one intelligent device is allowed. This capability allows the resident to simply integrate more intelligent sensors in the room without any adjustments of the system. The system automatically detects the included devices and starts to use them for environmental control. Within included BDF agents, the system scalability and the benefits to avoid single point of failure have been achieved. Additionally, BDF agents simplify the filtering and discovery of agents by their location and provided services.

From the side of decision making, three algorithms based on ANN, Fuzzy logic and Bayesian classifier have been explored. ANN based solution can produce good control results if resident's habits are permanent. In other case, the transition to new decisions usually is carried out step by step providing interim lighting control for resident during adaptation period. However, if resident requires a quick response to the changing environment and assumes that any actual change in the environment is significant, the intelligent lighting control based on FL or Bayes approach should be chosen which can provide more "strict" control solutions according to that latest resident's actions.

The results of the assessment of the times spent in various layers of the system show that duration of each control is mostly affected by Sensor Agents. So, the main attention should be given to the techniques of reducing time of sensors' needed for information gathering, processing and synchronization, as well as for facilitation of agents' communication.

\section{ACKNOWLEDGMENT}

This research was supported by the EU Structural Funds project VP1- 3.1-SMM-10-V-02-020 "Research on Smart Home Environment and Development of Intelligent 
Technologies (BIATech)".

\section{REFERENCES}

[1] I. Fatima, M. Fahim, Y. K. Lee, and S. Lee, "A unified framework for activity recognition-based behavior analysis and action prediction in smart homes," Sensors, vol. 13, no. 2, pp. 2682-2699, February 2013.

[2] M. Zhang and A. A. Sawchuk, "A feature selection-based framework for human activity recognition using wearable multimodal sensors," in Proc. the 6th International Conference on Body Area Networks, pp. 92-98. November 2011.

[3] O. Brdiczka, M. Langet, J. Maisonnasse, and J. L. Crowley "Detecting human behavior modelsfrom multimodal observation in a smart home," IEEE Transaction, Automation Science and Engine, vol. 6, no. 4, pp. 588-597, December 2009

[4] F. Iglesias Vazquez, and W. Kastner "A global approach of habit profiles for smart home control," in Proc. 13th Conference of International Building Performance Simulation Association, pp. 1969-1976, August 2013

[5] C. Reinisch, M. J. Kofler, F. I. Vazquez, and W. Kastner, "Think home energy efficiency in future smart homes," EURASIP Journal on Embedded Systems, no. 1, January 2011.

[6] T. A. Nguyen and M. Aiello, "Energy intelligent buildings based on user activity: A survey," Energy and Buildings, vol. 56, pp. 244-257, January 2013.

[7] C. Carrillo, E. D. Doradoa, J. Cidrasa, A. B. Pregala, P. Falconb, A. Fernandezc, and A. A. Sanchez, "Lighting control system based on digital camera for energy saving in shop windows," Energy and Buildings, vol. 59, pp. 143-151, December 2013.

[8] Z. Wang and Y. K. Tan, "Illumination control of LED systems based on neural network model and energy optimization algorithm," Energy and Buildings, vol. 62, pp. 514-521, July 2013.

[9] A. A. N. Kumaar, G. Kiran, and T. Sudarshan, " Intelligent lighting system using wireless sensor networks," International Journal of Ad hoc, Sensor \& Ubiquitous Computing, vol. 1, no. 4, pp. 17-27, December 2010

[10] L. Guo, M. Eloholma, and L. Halonen, "Lighting control strategies for telemanagement road lighting control systems," Journal of the Illuminating Engineering Society of North America (LEUKOS), vol. 4, no. 3, pp. 157-171, September 2013.

[11] P. M. Ferreira, A. E. Ruano, S. S. Silva, and E. Z. E. Conceicao, "Neural Networks based predictive control for thermal comfort and energy savings in public buildings," Energy and Buildings, vol. 55, pp. 238-251, December 2012.

[12] H. Medjahed, D. Istrate, J. Boudy, J. L. Baldinger, L. Bougueroua, M A. Dhouib, and B. Dorizzi. (2014). A Fuzzy Logic Approach for Remote Healthcare Monitoring by Learning and Recognizing Human Activities of Daily Living. Fuzzy Logic - Emerging Technologies and Applications. [Online], http://www.doc88.com/p-291365774121.htm

[13] O. Brdiczka, J. Maisonnasse, P. Reignier, and J. L. Crowley, "Learning individual roles from video in a smart home," presented at the 2nd IET International Conference, Athens, Greece, July 5 - 6, 2006.

[14] R. Abaalkhail, M. Orozco, and A. El Saddik, "Home energy saving for heating/cooling system by distributed intelligent energy controller," presented at the Instrumentation and Measurement Technology Conference in Graz, pp. 604-609, May 13 - 16, 2012.

[15] A. Rogers, S. D. Ramchurn, and N. R. Jennings, "Delivering the Smart Grid: Challenges for Autonomous Agents and Multi-Agent Systems Research," presented at the Conference on Artificial Intelligence in Toronto, Canada, 22 - 26 Jul, 2012.

[16] D. Cavone, B. D. Carolis, S. Ferilli, and N. Novielli, "An agent-based approach for adapting the behavior of a smart home environment," presented at the 12th Workshop on Objects and Agents in Italy, Jul 4 -6, 2011.

[17] A. E. F. Seghrouchni, A. Olaru, T. T. N. Nguyen, and D. Salomone, "Agent oriented design for ambient intelligence," presented at the Principles and Practice of Multi-Agent Systems in Kolkata, India, November 12-15, 2010.

[18] F. Bellifeminee, A. Poggi, and G. Rimassa, "JADE: A FIPA2000 compliant agent development environment," presented at the
Proceedings of the fifth international conference on Autonomous agents in Canada, May 28 - June 02, 2001.

[19] S. Poslad, "Specifying protocols for multi-agent system interaction," ACM Transactions on Autonomous and Adaptive Systems (TAAS), vol. 2, no. 4, pp. 24, November 2007.

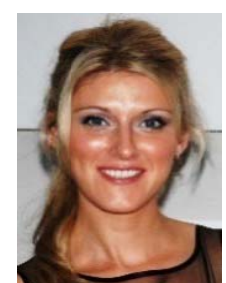

Agne Paulauskaite-Taraseviciene is an associate professor at the Department of Applied Informatics, and researcher at the Real-time Computer Systems Centre at Kaunas University of Technology. Her main research interests are formal specification, simulation and verification of dynamic structure adaptive systems, real time systems and theory of algorithms. Since 2007, she is constantly participating in research and development projects in the area of computation intelligence in human-computer interaction, assistive technology and robotics and smart environment.

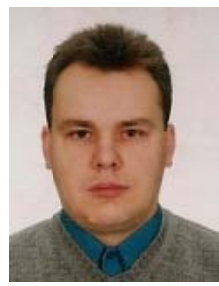

Nerijus Morkevicius is an associated professor of the Kaunas University of Technology (KTU). He received the $\mathrm{PhD}$ in computer science in Kaunas University of Technology department of Applied Mathematics in 2002. He has taken part in several national and EU funded research projects, has a number of publications in high impact international conferences and journals. His main research interests are digital image processing, information management systems, enterprise application integration and intelligent control.

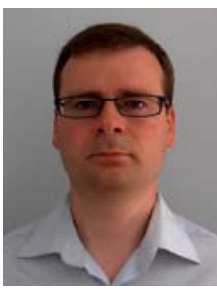

Vaidas Jukavicius is a $\mathrm{PhD}$ student and an assistant at the Department of Computer Science as well as a researcher at the Real-Time Computer Systems Centre at Kaunas University of Technology. Currently he is participating in research projects in the area of intelligent environment. His main research interests are digital signal processing, adaptive and real time systems, intelligent control and smart environment.

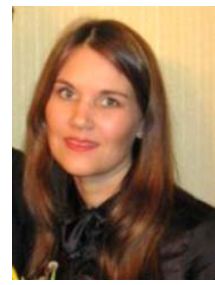

Laura Kizauskiene is an associate professor at the Department of Computers, and a researcher at the Real-Time Computer Systems Centre at Kaunas University of Technology. She has gained doctors' degree in technology science in 2010. Her main research interests are multi-agent systems, artificial intelligence, embedded real time systems and smart environments. Since 2007, she has been working at Kaunas University of Technology and has participated in several research projects, developing smart house and sensor network technologies.

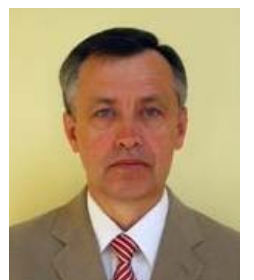

Egidijus Kazanavicius is the director of Real Time Computer Systems Centre. He is a board member of the Faculty of Informatics at Kaunas University of Technology, a professor at the Department of Computer Science at Kaunas University of Technology and he is a member of Euro group, Partnership for Innovation, a scientific coordinator of National Technologies Platform Network and Electronic Media. He is a scientific coordinator of international and national scientific projects. His main research interests are related with methodology for RTS design and simulation, formal models and design methodology for development of embedded software, devices and wireless sensor networks for smart home energy efficiency control, digital content distribution and management, internet of things, smart environment technologies. 